\title{
Two-Dimension Riemann Initial-Boundary Value Problem of Scalar Conservation Laws with Curved Boundary
}

\author{
Huazhou Chen ${ }^{1,2}$ and Tao Pan ${ }^{2}$ \\ ${ }^{1}$ Department of Mathematics, Shanghai University, Shanghai 200444, China \\ ${ }^{2}$ Key Laboratory of Optoelectronic Information and Sensing Technologies of Guangdong Higher Educational \\ Institutes, Jinan University, Guangzhou 510632, China
}

Correspondence should be addressed to Tao Pan, tpan@jnu.edu.cn

Received 16 December 2010; Accepted 1 February 2011

Academic Editor: Julio Rossi

Copyright (C) 2011 H. Chen and T. Pan. This is an open access article distributed under the Creative Commons Attribution License, which permits unrestricted use, distribution, and reproduction in any medium, provided the original work is properly cited.

\begin{abstract}
This paper is concerned with the structure of the weak entropy solutions to two-dimension Riemann initial-boundary value problem with curved boundary. Firstly, according to the definition of weak entropy solution in the sense of Bardos-Leroux-Nedelec (1979), the necessary and sufficient condition of the weak entropy solutions with piecewise smooth is given. The boundary entropy condition and its equivalent formula are proposed. Based on Riemann initial value problem, weak entropy solutions of Riemann initial-boundary value problem are constructed, the behaviors of solutions are clarified, and we focus on verifying that the solutions satisfy the boundary entropy condition. For different Riemann initial-boundary value data, there are a total of five different behaviors of weak entropy solutions. Finally, a worked-out specific example is given.
\end{abstract}

\section{Introduction}

Multidimensional conservation laws are a famous hard problem that plays an important role in mechanics and physics [1-3]. For Cauchy problem of multi-dimensional scalar conservation laws, Conway and Smoller [4] and Kruzkov [1] have proved that weak solution uniquely exists if it also satisfies entropy condition, and it is called weak entropy solutions. In order to further understand qualitative behavior of solutions, it is also important to investigate multi-dimensional Riemann problems. For two-dimensional case, Lindquist [5], Wagner [6], Zhang and Zheng [7] Guckenheimer [8], Zheng [9] among others, have discussed some relating Riemann problems. In a previous discussion, initial value contains several constant states with discontinuity lines so that self-similar transformations can be applied to reduce two-dimensional problem to one-dimensional case. The situation that 
initial value contains two constant states divided by a curve can not be solved by selfsimilar transformations, and Yang [10] proposed a new approach for construction of shock wave and rarefaction wave solutions; especially, rarefaction wave was got by constructing implicit function instead of the usual selfsimilar method. This approach can be expanded to general $n$ dimension. In addition, multi-dimensional scalar conservation laws with boundary are more common in practical problems. Bardos et al. [2] have proved the existence and uniqueness of the weak entropy solution of initial-boundary problems of multi-dimensional scalar conservation laws. The main difficulty for nonlinear conservation laws with boundary is to have a good formation of the boundary condition. Namely, for a fixed initial value, we really can not impose such a condition at the boundary, and the boundary condition is necessarily linked to the entropy condition. Moreover the behavior of solutions for one-dimensional problem with boundary was discussed in [11-18]. However, for multi-dimensional problem with boundary, the behaviors of solutions are still hard to study.

In this paper, two-dimensional case as an example of Yang's multi-dimensional Riemann problem [10] is expanded to the case with boundary. Considering two-dimensional Riemann problem for scalar conservation laws with curved boundary,

$$
\begin{gathered}
u_{t}+\frac{\partial f_{1}(u)}{\partial x_{1}}+\frac{\partial f_{2}(u)}{\partial x_{2}}=0, \quad\left(x_{1}, x_{2}\right) \in \Omega, t>0, \\
\left.u\right|_{t=0}=u_{+}, \quad\left(x_{1}, x_{2}\right) \in \Omega, \\
\left.u\right|_{\Gamma}=u_{-}, \quad t>0,
\end{gathered}
$$

where $u=u\left(t, x_{1}, x_{2}\right), u_{+}$and $u_{-}$are both constants, $f_{1}(u), f_{2}(u) \in C^{2}(R), M\left(x_{1}, x_{2}\right) \in C^{1}\left(R^{2}\right)$, $M\left(x_{1}, x_{2}\right)=0$ is a smooth manifold and divides $R^{2}$ into two infinite parts, $\Omega=\left\{\left(x_{1}, x_{2}\right) \mid\right.$ $\left.M\left(x_{1}, x_{2}\right)>0\right\}$, and $\Gamma=\left\{\left(t, x_{1}, x_{2}\right) \mid M\left(x_{1}, x_{2}\right)=0, t>0\right\}$ and denote $\left.u\right|_{\Gamma}=\gamma u$.

In Section 2, weak entropy solution of Riemann initial-boundary value problem (1.1) is defined, and the boundary entropy condition is discussed. In Section 3, weak entropy solutions of the corresponding Riemann initial value problem are expressed. In Section 4, using the weak entropy solutions of the corresponding Riemann initial value problem, we construct the weak entropy solutions of Riemann initial-boundary value problem, and prove that they satisfy the boundary entropy condition. The weak entropy solutions include a total of five different shock and rarefaction wave solutions based on different Riemann data. Finally, in Section 5, we give a worked-out specific example.

\section{Preliminaries}

According to the definition of the weak entropy solution and the boundary entropy condition to the general initial-boundary problems of multi-dimensional scalar conservation laws which was proposed by Bardos et al. [2] and Pan and Lin [13], we can obtain the following definition and three lemmas for Riemann initial-boundary value problem (1.1).

Definition 2.1. A locally bounded and bounded variation function $u\left(t, x_{1}, x_{2}\right)$ on $(0,+\infty) \times \Omega$ is called a weak entropy solution of Riemann initial-boundary value problem (1.1) if, for any real constant $k$ and for any nonnegative function $\varphi\left(t, x_{1}, x_{2}\right) \in C_{0}^{\infty}((0,+\infty) \times \Omega)$, it satisfies 
the following inequality:

$$
\begin{aligned}
& \int_{0}^{+\infty} \int_{M>0}\left(|u-k| \varphi_{t}+\operatorname{sgn}(u-k)\left(f_{1}(u)-f_{1}(k)\right) \varphi_{x_{1}}+\operatorname{sgn}(u-k)\left(f_{2}(u)-f_{2}(k)\right) \varphi_{x_{2}}\right) \\
& \quad \times d x_{1} d x_{2} d t+\int_{M>0}\left|u_{+}-k\right| \varphi\left(0, x_{1}, x_{2}\right) d x_{1} d x_{2} \\
& \quad+\int_{\Gamma} \operatorname{sgn}\left(u_{-}-k\right)\left(f_{1}(\gamma u)-f_{1}(k), f_{2}(\gamma u)-f_{2}(k)\right) \circ \vec{n} \gamma \varphi d x_{1} d x_{2} d t \geq 0,
\end{aligned}
$$

where $\vec{n}$ is the outward normal vector of curve $M\left(x_{1}, x_{2}\right)$.

Lemma 2.2. If $u\left(t, x_{1}, x_{2}\right)$ is a weak entropy solution of (1.1), then it satisfies the following boundary: entropy condition

$$
\operatorname{sgn}\left(\gamma u-u_{-}\right)\left(f_{1}(\gamma u)-f_{1}(k), f_{2}(\gamma u)-f_{2}(k)\right) \circ \vec{n} \geq 0, \quad k \in I\left(\gamma u, u_{-}\right), \text {a.e. } t>0,
$$

where $I\left(\gamma u, u_{-}\right)=\left[\min \left(\gamma u, u_{-}\right), \max \left(\gamma u, u_{-}\right)\right]$.

It can be easily proved that $\forall k \in I\left(\gamma u, u_{-}\right), \operatorname{sgn}\left(\gamma u-u_{-}\right)=\operatorname{sgn}(\gamma u-k)$, so (2.2) can be rewritten as

$$
\operatorname{sgn}(\gamma u-k)\left(f_{1}(\gamma u)-f_{1}(k), f_{2}(\gamma u)-f_{2}(k)\right) \circ \vec{n} \geq 0, \quad k \in I\left(\gamma u, u_{-}\right) \text {, a.e. } t>0,
$$

thus one can get $r u=u_{-}$or

$$
\vec{n} \circ\left(\frac{f_{1}(\gamma u)-f_{1}(k)}{\gamma u-k}, \frac{f_{2}(\gamma u)-f_{2}(k)}{\gamma u-k}\right) \geq 0, \quad k \in I\left(\gamma u, u_{-}\right), k \neq \gamma u \text {, a.e. } t>0,
$$

and one notices that $\vec{n}=\left(-M_{x_{1}},-M_{x_{2}}\right), M_{x_{1}}=\partial M\left(x_{1}, x_{2}\right) / \partial x_{1}, M_{x_{2}}=\partial M\left(x_{1}, x_{2}\right) / \partial x_{2}$, then boundary entropy condition (2.2) is equivalent to

$$
\begin{aligned}
& r u=u_{-} \text {or } \\
& M_{x_{1}} \frac{f_{1}(\gamma u)-f_{1}(k)}{\gamma u-k}+M_{x_{2}} \frac{f_{2}(\gamma u)-f_{2}(k)}{\gamma u-k} \leq 0, \quad k \in I\left(\gamma u, u_{-}\right), k \neq \gamma u, \text { a.e. } t>0 .
\end{aligned}
$$

The proof for one-dimension case of Lemma 2.2 can be found in Pan and Lin's work [13], and the proof for $n$-dimension case is totally similar to one-dimension case; actually the idea of the proof first appears in Bardos et al.'s work [2], so the proof details for Lemma 2.2 are omitted here.

Lemma 2.3. A piecewise smooth function $u\left(t, x_{1}, x_{2}\right)$ with smooth discontinuous surface is a weak entropy solution to the Riemann initial-boundary value problem (1.1) in the sense of (2.1) if and only if the following conditions are satisfied. 
(i) Rankine-Hugoniot condition: At any point $\mathrm{P}$ on discontinuity surface $\bar{S}$ of solution $u\left(t, x_{1}, x_{2}\right), \vec{N}_{\mathrm{P}}$ is a unit normal vector to $\bar{S}$ at $\mathrm{P}$ if

$$
\begin{aligned}
& u_{r}=\lim _{\varepsilon \rightarrow 0^{+}} u(P+\varepsilon n), \\
& u_{l}=\lim _{\varepsilon \rightarrow 0^{-}} u(P+\varepsilon n),
\end{aligned}
$$

then

$$
\vec{N}_{\mathrm{P}} \circ\left([u],\left[f_{1}\right],\left[f_{2}\right]\right)=0,
$$

where $[u]=u_{r}-u_{l},\left[f_{1}\right]=f_{1}\left(u_{r}\right)-f_{1}\left(u_{l}\right),\left[f_{2}\right]=f_{2}\left(u_{r}\right)-f_{2}\left(u_{l}\right)$.

For any constant $k \in\left[u_{l}, u_{r}\right], \mathrm{P} \in \bar{S}$,

$$
\vec{N}_{\mathrm{P}} \circ\left(k-u_{l}, f_{1}(k)-f_{1}\left(u_{l}\right), f_{2}(k)-f_{2}\left(u_{l}\right)\right) \geq 0
$$

or equivalently

$$
\vec{N}_{\mathrm{P}} \circ\left(k-u_{r}, f_{1}(k)-f_{1}\left(u_{r}\right), f_{2}(k)-f_{2}\left(u_{r}\right)\right) \geq 0 .
$$

(ii) Boundary entropy condition:

$$
\begin{gathered}
r u=u_{-} \text {or } \\
M_{x_{1}} \frac{f_{1}(\gamma u)-f_{1}(k)}{r u-k}+M_{x_{2}} \frac{f_{2}(\gamma u)-f_{2}(k)}{r u-k} \leq 0, \quad k \in I\left(\gamma u, u_{-}\right), k \neq \gamma u, \text { a.e. } t>0 .
\end{gathered}
$$

(iii) Initial value condition:

$$
u\left(0, x_{1}, x_{2}\right)=u_{0}\left(x_{1}, x_{2}\right), \quad M\left(x_{1}, x_{2}\right)>0 .
$$

For piecewise smooth solution with smooth discontinuous surface, Rankine-Hugoniot condition (2.7), entropy conditions (2.8), (2.9) and initial value condition (2.11) are obviously satisfied, see also the previous famous works in [4,7-9]. As in Lemma 2.2, boundary entropy condition (2.10) also holds. The proof of the converse in not difficult and is omitted here.

According to Bardos et al.'s work [2], we have the following Lemma.

Lemma 2.4. If $u\left(t, x_{1}, x_{2}\right)$ is piecewise smooth weak entropy solution of (1.1) which satisfies the conditions of Lemma 2.3 , then $u\left(t, x_{1}, x_{2}\right)$ is unique.

According to the uniqueness of weak entropy solution, as long as the piecewise smooth function satisfying Lemma 2.3 is constructed, the weak entropy solution of Riemann initialboundary value problem can be obtained. 


\section{Solution of Riemann Initial Value Problem}

First, we study the Riemann initial value problem corresponding to the Riemann initialboundary value problem (1.1) as follows:

$$
\begin{gathered}
u_{t}+\frac{\partial f_{1}(u)}{\partial x_{1}}+\frac{\partial f_{2}(u)}{\partial x_{2}}=0, \quad t>0, \\
\left.u\right|_{t=0}= \begin{cases}u_{-}, & M\left(x_{1}, x_{2}\right)<0 \\
u_{+}, & M\left(x_{1}, x_{2}\right)>0 .\end{cases}
\end{gathered}
$$

Condition $\mathscr{d}$ For $u \in(a, b)$,

$$
M_{x_{1}} f_{1}^{\prime \prime}(u)+M_{x_{2}} f_{2}^{\prime \prime}(u)>0,
$$

where $(a, b)$ is a certain interval $a, b$ can be a finite number or $\infty$.

Condition $\mathscr{d}$ combines flux functions $f_{1}, f_{2}$ and curved boundary manifold $M$, providing necessary condition for the convex property of the new flux function which will be constructed in formula (4.5). The convex property clarifies whether the characteristics intersect or not, whether the weak solution satisfied internal entropy conditions (2.8) and (2.9) and boundary entropy condition (2.10), In addition, Condition $\mathscr{H}$ is easily satisfied, for example, $f_{1}(u)=f_{2}(u)=(1 / 2) u^{2}, M\left(x_{1}, x_{2}\right)=x_{1}^{3}+x_{2}$, then $M_{x_{1}} f_{1}^{\prime \prime}(u)+M_{x_{2}} f_{2}^{\prime \prime}(u)=3 x_{1}^{2}+1>0$, so Condition $\mathscr{t}$ holds. Here $M\left(x_{1}, x_{2}\right)=0$ is a cubic curve on the $X_{1}-X_{2}$ plane, and it is strictly bending.

Yang's work [10] showed that depending on whether the characteristics intersect or not, the weak entropy solution of (3.1) has two forms as follows.

Lemma 3.1 (see [10]). Suppose ( $\mathscr{d}$ ) holds. If $\boldsymbol{u}_{-}>\boldsymbol{u}_{+}$, then weak entropy solution of (3.1) is shock wave solution $\mathcal{S}$, and

$$
u\left(t, x_{1}, x_{2}\right)= \begin{cases}u_{-}, & M\left(x_{1}-\frac{\left[f_{1}\right]}{[u]} t, x_{2}-\frac{\left[f_{2}\right]}{[u]} t\right)<0, \\ u_{+}, & M\left(x_{1}-\frac{\left[f_{1}\right]}{[u]} t, x_{2}-\frac{\left[f_{2}\right]}{[u]} t\right)>0,\end{cases}
$$

and discontinuity surface $\bar{S}\left(t, x_{1}, x_{2}\right)=0$ is

$$
M\left(x_{1}-\frac{\left[f_{1}\right]}{[u]} t, x_{2}-\frac{\left[f_{2}\right]}{[u]} t\right)=0
$$

where $[u]=u_{+}-u_{-},\left[f_{1}\right]=f_{1}\left(u_{+}\right)-f_{1}\left(u_{-}\right),\left[f_{2}\right]=f_{2}\left(u_{+}\right)-f_{2}\left(u_{-}\right)$. 
Lemma 3.2 (see [10]). Suppose that ( $\mathscr{C})$ holds. If $\boldsymbol{u}_{-}<\boldsymbol{u}_{+}$, then weak entropy solution of (3.1) is rarefaction wave solution $R$, and

$$
u\left(t, x_{1}, x_{2}\right)=\left\{\begin{array}{lll}
u_{-}, & 0>M\left(x_{1}-f_{1}^{\prime}\left(u_{-}\right) t, x_{2}-f_{2}^{\prime}\left(u_{-}\right) t\right), & t>0, \\
C\left(t, x_{1}, x_{2}\right), & M\left(x_{1}-f_{1}^{\prime}\left(u_{-}\right) t, x_{2}-f_{2}^{\prime}\left(u_{-}\right) t\right) \geq 0 \\
& \geq M\left(x_{1}-f_{1}^{\prime}\left(u_{+}\right) t, x_{2}-f_{2}^{\prime}\left(u_{+}\right) t\right), & t>0, \\
u_{+}, & M\left(x_{1}-f_{1}^{\prime}\left(u_{+}\right) t, x_{2}-f_{2}^{\prime}\left(u_{+}\right) t\right)>0, & t>0,
\end{array}\right.
$$

where $C\left(t, x_{1}, x_{2}\right)$ is the implicit function which satisfies

$$
M\left(x_{1}-f_{1}^{\prime}(C) t, x_{2}-f_{2}^{\prime}(C) t\right)=0 .
$$

Theorem 3.3 (see [10]). Suppose that ( $\mathscr{\ell}$ ) holds. Given $u_{-}, u_{+} \in(a, b)$, then

(i) if $u_{-}>u_{+}$, weak entropy solution of (3.1) is $\mathcal{S}$ and $u\left(t, x_{1}, x_{2}\right)$ has a form as (3.3);

(ii) if $u_{-}<u_{+}$, weak entropy solution of (3.1) is $\mathcal{R}$ and $u\left(t, x_{1}, x_{2}\right)$ has a form as (3.5);

(iii) weak entropy solutions formed as (3.3) and (3.5) uniquely exist.

The weak entropy solutions constructed here are piecewise smooth and satisfy conditions (i) and (iii) of Lemma 2.3.

\section{Solution of Riemann Initial-Boundary Value Problem}

Now we restrict the weak entropy solutions of the Riemann initial value problem (3.1) constructed in Section 3 in region $\{t>0\} \times \Omega$, and they still satisfy conditions (i) and (iii) of Lemma 2.3. If they also satisfy the boundary entropy condition (ii) of Lemma 2.3, then they are the weak entropy solutions of Riemann initial-boundary value problem (1.1).

Based on different Riemann data of $u_{+}$and $u_{-}$, the weak entropy solutions of the Riemann initial value problem (3.1) have the following five different behaviors when restricted in region $\{t>0\} \times \Omega$.

If $u_{-}>u_{+}$, the solution of (3.1) is shock wave $\mathcal{S}$ and

$$
u\left(t, x_{1}, x_{2}\right)=\left\{\begin{array}{l}
u_{-}, \quad M\left(x_{1}-\frac{\left[f_{1}\right]}{[u]} t, x_{2}-\frac{\left[f_{2}\right]}{[u]} t\right)<0, \quad M\left(x_{1}, x_{2}\right)>0, t>0, \\
u_{+}, \quad M\left(x_{1}-\frac{\left[f_{1}\right]}{[u]} t, x_{2}-\frac{\left[f_{2}\right]}{[u]} t\right)>0, \quad M\left(x_{1}, x_{2}\right)>0, t>0 .
\end{array}\right.
$$

$M\left(x_{1}-\left(\left[f_{1}\right] /[u]\right) t, x_{2}-\left(\left[f_{2}\right] /[u]\right) t\right)=0$ is formed by moving $M\left(x_{1}, x_{2}\right)=0$ along the direction of the vector $\left(\left[f_{1}\right] /[u],\left[f_{2}\right] /[u]\right)=\left(\left(f_{1}\left(u_{+}\right)-f_{1}\left(u_{-}\right)\right) /\left(u_{+}-u_{-}\right),\left(f_{2}\left(u_{+}\right)-\right.\right.$ $\left.\left.f_{2}\left(u_{-}\right)\right) /\left(u_{+}-u_{-}\right)\right) \triangleq \vec{\alpha}$, and the outward normal vector $\vec{n}$ of curve $M\left(x_{1}, x_{2}\right)=0$ is equal to $\left(-M_{x_{1}},-M_{x_{2}}\right)$. According to the angle between $\vec{\alpha}$ and $\vec{n}$, the solution restricted in $\{t>0\} \times \Omega$ has two behaviors as follows.

Case 1. If $u_{-}>u_{+}$and $\vec{n} \circ\left(\left[f_{1}\right] /[u],\left[f_{2}\right] /[u]\right) \geq 0$. 


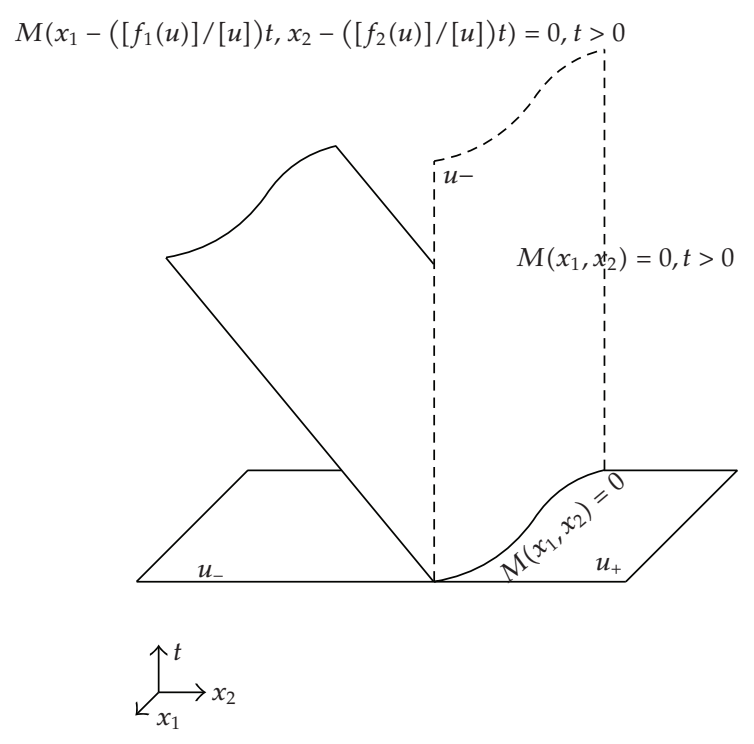

(a) The constant solution

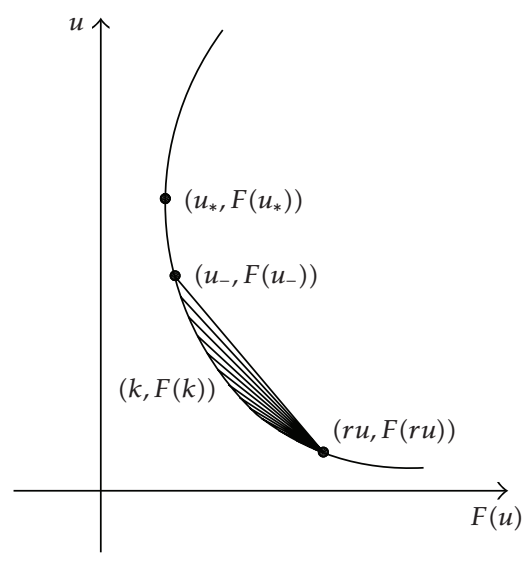

(b) The phase plane $(u, F(u))$

Figure 1: Case 1.

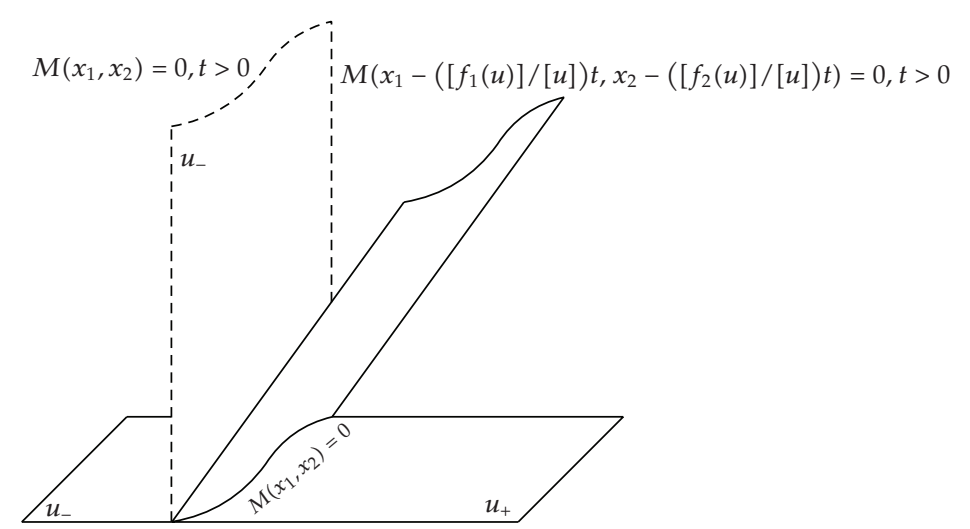

$$
\overbrace{x_{1}}^{t} x_{2}
$$

Figure 2: The shock wave solution of Case 2.

See also Figure 1(a); it shows that the angle between $\vec{\alpha}$ and $\vec{n}$ is an acute angle, the shock wave surface $M\left(x_{1}-\left(\left[f_{1}\right] /[u]\right) t, x_{2}-\left(\left[f_{2}\right] /[u]\right) t\right)=0$ is outside region $\{t>0\} \times \Omega$, and the solution is constant state formed as

$$
u\left(t, x_{1}, x_{2}\right)=u_{+}, \quad M\left(x_{1}, x_{2}\right) \geq 0, \quad t>0 .
$$

Case 2. If $u_{-}>u_{+}$and $\vec{n} \circ\left(\left[f_{1}\right] /[u],\left[f_{2}\right] /[u]\right)<0$. 
See also Figure 2; it shows that the angle between $\vec{\alpha}$ and $\vec{n}$ is an obtuse angle, the shock wave surface $M\left(x_{1}-\left(\left[f_{1}\right] /[u]\right) t, x_{2}-\left(\left[f_{2}\right] /[u]\right) t\right)=0$ is inside region $\{t>0\} \times \Omega$, and the solution is shock wave formed as

$$
u\left(t, x_{1}, x_{2}\right)=\left\{\begin{array}{l}
u_{-}, \quad M\left(x_{1}, x_{2}\right)>0>M\left(x_{1}-\frac{\left[f_{1}\right]}{[u]} t, x_{2}-\frac{\left[f_{2}\right]}{[u]} t\right), \quad t>0 \\
u_{+}, \quad M\left(x_{1}-\frac{\left[f_{1}\right]}{[u]} t, x_{2}-\frac{\left[f_{2}\right]}{[u]} t\right)>0, \quad t>0 .
\end{array}\right.
$$

If $u_{-}<u_{+}$, the solution of (3.1) is rarefaction wave $R$ and

$$
u\left(t, x_{1}, x_{2}\right)= \begin{cases}u_{-}, & M\left(x_{1}-f_{1}^{\prime}\left(u_{-}\right) t, x_{2}-f_{2}^{\prime}\left(u_{-}\right) t\right)<0, \\ & M\left(x_{1}, x_{2}\right)>0, \quad t>0, \\ C\left(t, x_{1}, x_{2}\right), & M\left(x_{1}-f_{1}^{\prime}\left(u_{-}\right) t, x_{2}-f_{2}^{\prime}\left(u_{-}\right) t\right) \geq 0, \\ & M\left(x_{1}-f_{1}^{\prime}\left(u_{+}\right) t, x_{2}-f_{2}^{\prime}\left(u_{+}\right) t\right) \leq 0, \\ & M\left(x_{1}, x_{2}\right)>0, \quad t>0 \\ & M\left(x_{1}-f_{1}^{\prime}\left(u_{+}\right) t, x_{2}-f_{2}^{\prime}\left(u_{+}\right) t\right)>0, \\ & M\left(x_{1}, x_{2}\right)>0, \quad t>0 .\end{cases}
$$

$M\left(x_{1}-f_{1}^{\prime}\left(u_{-}\right) t, x_{2}-f_{2}^{\prime}\left(u_{-}\right) t\right)=0$ is formed by moving $M\left(x_{1}, x_{2}\right)=0$ along the direction of the vector $\left(f_{1}^{\prime}\left(u_{-}\right), f_{2}^{\prime}\left(u_{-}\right)\right) \triangleq \overrightarrow{\beta_{-}}, M\left(x_{1}-f_{1}^{\prime}\left(u_{+}\right) t, x_{2}-f_{2}^{\prime}\left(u_{+}\right) t\right)=0$ is formed by moving $M\left(x_{1}, x_{2}\right)=0$ along the direction of the vector $\left(f_{1}^{\prime}\left(u_{+}\right), f_{2}^{\prime}\left(u_{+}\right)\right) \triangleq \vec{\beta}_{+}$, and the outward normal vector $\vec{n}$ of curve $M\left(x_{1}, x_{2}\right)=0$ is equal to $\left(-M_{x_{1}},-M_{x_{2}}\right)$.

We construct a new flux function

$$
f\left(x_{1}, x_{2}, u\right)=M_{x_{1}} f_{1}(u)+M_{x_{2}} f_{2}(u) \triangleq F(u),
$$

according to condition $(\mathscr{\ell}), F^{\prime \prime}(u)=M_{x_{1}} f_{1}^{\prime \prime}(u)+M_{x_{2}} f_{2}^{\prime \prime}(u)>0, F(u)$ is convex, and $F^{\prime}(u)$ is monotonically increasing function, so $F^{\prime}\left(u_{-}\right)<F^{\prime}\left(u_{+}\right)$. And also

$$
\begin{aligned}
& F^{\prime}\left(u_{-}\right)=M_{x_{1}} f_{1}^{\prime}\left(u_{-}\right)+M_{x_{2}} f_{2}^{\prime}\left(u_{-}\right)=-\vec{n} \circ\left(f_{1}^{\prime}\left(u_{-}\right), f_{2}^{\prime}\left(u_{-}\right)\right), \\
& F^{\prime}\left(u_{+}\right)=M_{x_{1}} f_{1}^{\prime}\left(u_{+}\right)+M_{x_{2}} f_{2}^{\prime}\left(u_{+}\right)=-\vec{n} \circ\left(f_{1}^{\prime}\left(u_{+}\right), f_{2}^{\prime}\left(u_{+}\right)\right) .
\end{aligned}
$$

Thus, $\vec{n} \circ\left(f_{1}^{\prime}\left(u_{-}\right), f_{2}^{\prime}\left(u_{-}\right)\right)>\vec{n} \circ\left(f_{1}^{\prime}\left(u_{+}\right), f_{2}^{\prime}\left(u_{+}\right)\right)$. According to the angles between $\overrightarrow{\beta_{+}}, \overrightarrow{\beta_{-}}$, and $\vec{n}$, the solution restricted in $\{t>0\} \times \Omega$ has three behaviors as follows.

Case 3. If $u_{-}<u_{+}$and $\vec{n} \circ\left(f_{1}^{\prime}\left(u_{+}\right), f_{2}^{\prime}\left(u_{+}\right)\right)<\vec{n} \circ\left(f_{1}^{\prime}\left(u_{-}\right), f_{2}^{\prime}\left(u_{-}\right)\right) \leq 0$. 


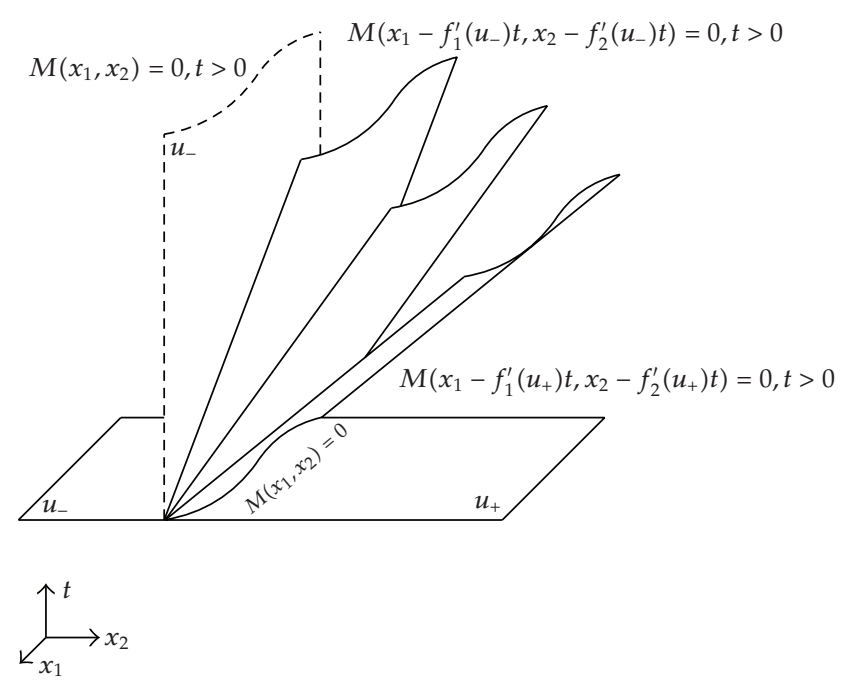

Figure 3: The rarefaction wave solution of Case 3.

See also Figure 3 ; it shows that the angles between $\overrightarrow{\beta_{+}}, \overrightarrow{\beta_{-}}$, and $\vec{n}$ are obtuse angles, the rarefaction wave surfaces $M\left(x_{1}-f_{1}^{\prime}\left(u_{+}\right) t, x_{2}-f_{2}^{\prime}\left(u_{+}\right) t\right)=0$ and $M\left(x_{1}-f_{1}^{\prime}\left(u_{-}\right) t, x_{2}-f_{2}^{\prime}\left(u_{-}\right) t\right)=0$ are both inside region $\{t>0\} \times \Omega$, and the solution is rarefaction wave formed as

$$
u\left(t, x_{1}, x_{2}\right)= \begin{cases}u_{-,} & M\left(x_{1}, x_{2}\right)>0>M\left(x_{1}-f_{1}^{\prime}\left(u_{-}\right) t, x_{2}-f_{2}^{\prime}\left(u_{-}\right) t\right), \quad t>0, \\ C\left(t, x_{1}, x_{2}\right), & M\left(x_{1}-f_{1}^{\prime}\left(u_{-}\right) t, x_{2}-f_{2}^{\prime}\left(u_{-}\right) t\right) \geq 0 \\ & \geq M\left(x_{1}-f_{1}^{\prime}\left(u_{+}\right) t, x_{2}-f_{2}^{\prime}\left(u_{+}\right) t\right), \quad t>0, \\ u_{+}, & M\left(x_{1}-f_{1}^{\prime}\left(u_{+}\right) t, x_{2}-f_{2}^{\prime}\left(u_{+}\right) t\right)>0, \quad t>0,\end{cases}
$$

where $C\left(t, x_{1}, x_{2}\right)$ is the implicit function which satisfies (3.6).

Case 4. If $u_{-}<u_{+}$and $\vec{n} \circ\left(f_{1}^{\prime}\left(u_{+}\right), f_{2}^{\prime}\left(u_{+}\right)\right)<0<\vec{n} \circ\left(f_{1}^{\prime}\left(u_{-}\right), f_{2}^{\prime}\left(u_{-}\right)\right)$.

See also Figure $4(\mathrm{a})$; it shows that the angle between $\overrightarrow{\beta_{+}}$and $\vec{n}$ is an obtuse angle, the angle between $\overrightarrow{\beta_{-}}$and $\vec{n}$ is an acute angles, the rarefaction wave surface $M\left(x_{1}-f_{1}^{\prime}\left(u_{+}\right) t, x_{2}-\right.$ $\left.f_{2}^{\prime}\left(u_{+}\right) t\right)=0$ is inside region $\{t>0\} \times \Omega$, the rarefaction wave surface $M\left(x_{1}-f_{1}^{\prime}\left(u_{-}\right) t, x_{2}-\right.$ $\left.f_{2}^{\prime}\left(u_{-}\right) t\right)=0$ is outside region $\{t>0\} \times \Omega$, and the solution is rarefaction wave formed as

$$
u\left(t, x_{1}, x_{2}\right)= \begin{cases}C\left(t, x_{1}, x_{2}\right), & M\left(x_{1}, x_{2}\right)>0 \geq M\left(x_{1}-f_{1}^{\prime}\left(u_{+}\right) t, x_{2}-f_{2}^{\prime}\left(u_{+}\right) t\right), \quad t>0, \\ u_{+}, & M\left(x_{1}-f_{1}^{\prime}\left(u_{+}\right) t, x_{2}-f_{2}^{\prime}\left(u_{+}\right) t\right)>0, \quad t>0,\end{cases}
$$

where $C\left(t, x_{1}, x_{2}\right)$ is the implicit function which satisfies (3.6).

Case 5. If $u_{-}<u_{+}$and $0 \leq \vec{n} \circ\left(f_{1}^{\prime}\left(u_{+}\right), f_{2}^{\prime}\left(u_{+}\right)\right)<\vec{n} \circ\left(f_{1}^{\prime}\left(u_{-}\right), f_{2}^{\prime}\left(u_{-}\right)\right)$. 


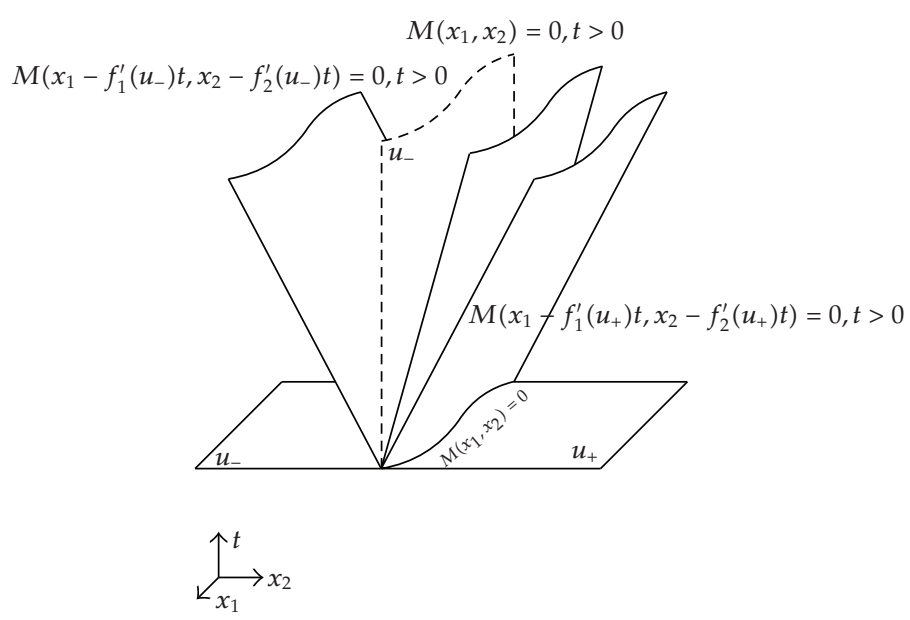

(a) The rarefaction wave solution

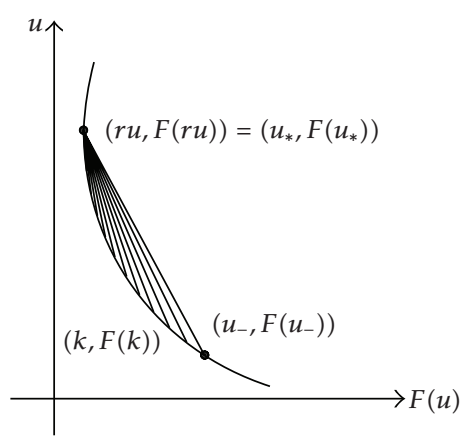

(b) The phase plane $(u, F(u))$

Figure 4: Case 4.

See also Figure $5(\mathrm{a})$; it shows that the angles between $\vec{\beta}_{+}, \vec{\beta}_{-}$, and $\vec{n}$ are acute angles, the rarefaction wave surfaces $M\left(x_{1}-f_{1}^{\prime}\left(u_{+}\right) t, x_{2}-f_{2}^{\prime}\left(u_{+}\right) t\right)=0$ and $M\left(x_{1}-f_{1}^{\prime}\left(u_{-}\right) t, x_{2}-f_{2}^{\prime}\left(u_{-}\right) t\right)=0$ are both outside region $\{t>0\} \times \Omega$, and the solution is constant state formed as

$$
u\left(t, x_{1}, x_{2}\right)=u_{+}, \quad M\left(x_{1}, x_{2}\right)>0, \quad t>0 .
$$

Next, we verify the above five solutions all satisfying the boundary entropy condition (ii) of Lemma 2.3. By noticing the definition of $F(u)$ (4.5) and its convex property, the boundary entropy condition (ii) of Lemma 2.3 can be equivalent to the following formula

$$
\gamma u=u_{-} \text {or } \frac{F(\gamma u)-F(k)}{\gamma u-k} \leq 0, \quad k \in I\left(\gamma u, u_{-}\right), k \neq \gamma u \text {, a.e. } t>0,
$$

and thus we verify the above five solutions all satisfying the boundary entropy condition (4.10).

Case 1. When $u_{-}>u_{+}, \vec{n} \circ\left(\left[f_{1}\right] /[u],\left[f_{2}\right] /[u]\right) \geq 0$, the shock wave solution is formed as (4.2). In this case, $\gamma u=u_{+} \neq u_{-}$since

$$
\left.\frac{F(\gamma u)-F\left(u_{-}\right)}{\gamma u-u_{-}}=-\vec{n} \circ\left(\frac{\left[f_{1}\right]}{[u]}, \frac{\left[f_{2}\right]}{[u]}\right) \leq 0 \text { (see also Figure } 1(\mathrm{~b})\right),
$$

and $\gamma u<u_{-} \leq u_{*}$, where $u_{*}$ is the extreme point of $F(u)$. For $\forall k \in\left[\gamma u, u_{-}\right]$, according to the convex property of $F(u)$, we have that

$$
\frac{F(r u)-F(k)}{r u-k} \leq \frac{F(r u)-F\left(u_{-}\right)}{r u-u_{-}} \leq 0,
$$

and so the boundary entropy condition (4.10) is verified. 

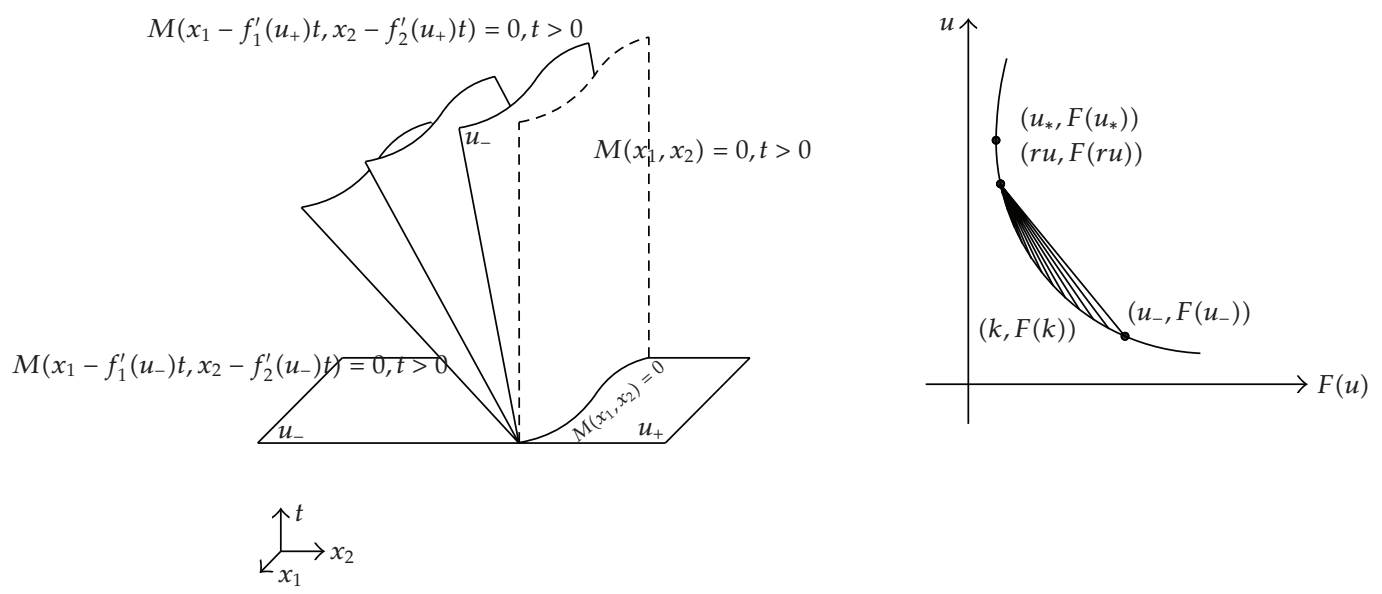

(a) The constant solution

(b) The phase plane $(u, F(u))$

Figure 5: Case 5.

Case 2. When $u_{-}>u_{+}, \vec{n} \circ\left(\left[f_{1}\right] /[u],\left[f_{2}\right] /[u]\right)<0$, the shock wave solution is formed as (4.3). In this case, $\gamma u=u_{-}$, so the boundary entropy condition (4.10) is naturally verified.

Case 3. When $u_{-}<u_{+}, \vec{n} \circ\left(f_{1}^{\prime}\left(u_{+}\right), f_{2}^{\prime}\left(u_{+}\right)\right)<\vec{n} \circ\left(f_{1}^{\prime}\left(u_{-}\right), f_{2}^{\prime}\left(u_{-}\right)\right) \leq 0$, the rarefaction wave solution is formed as (4.7). In this case, $\gamma u=u_{-}$, and so the boundary entropy condition (4.10) is naturally verified.

Case 4. When $u_{-}<u_{+}, \vec{n} \circ\left(f_{1}^{\prime}\left(u_{+}\right), f_{2}^{\prime}\left(u_{+}\right)\right)<0<\vec{n} \circ\left(f_{1}^{\prime}\left(u_{-}\right), f_{2}^{\prime}\left(u_{-}\right)\right)$, the rarefaction wave solution is formed as (4.8). In this case, $r u=\left.C\left(t, x_{1}, x_{2}\right)\right|_{M\left(x_{1}, x_{2}\right)=0} \triangleq u_{*} \leq u_{-}, F^{\prime}\left(u_{*}\right)=0$ and $F^{\prime}\left(u_{-}\right)=-\vec{n} \circ\left(f_{1}^{\prime}\left(u_{-}\right), f_{2}^{\prime}\left(u_{-}\right)\right)<0<-\vec{n} \circ\left(f_{1}^{\prime}\left(u_{+}\right), f_{2}^{\prime}\left(u_{+}\right)\right)=F^{\prime}\left(u_{+}\right)$(see also Figure 4(b)), namely, $F^{\prime}\left(u_{-}\right)<F^{\prime}(\gamma u)=0<F^{\prime}\left(u_{+}\right)$. For $\forall k \in\left[u_{-}, \gamma u\right]$, according to the convex property of $F(u)$ and Lagrange mean value theorem, there exists $\xi \in[k, \gamma u]$, satisfying

$$
\frac{F(r u)-F(k)}{r u-k}=F^{\prime}(\xi) \leq F^{\prime}(r u)=0,
$$

and so the boundary entropy condition (4.10) is verified.

Case 5. When $u_{-}<u_{+}, 0 \leq \vec{n} \circ\left(f_{1}^{\prime}\left(u_{+}\right), f_{2}^{\prime}\left(u_{+}\right)\right)<\vec{n} \circ\left(f_{1}^{\prime}\left(u_{-}\right), f_{2}^{\prime}\left(u_{-}\right)\right)$, the rarefaction wave solution is formed as (4.9). In this case, $\gamma u=u_{+}>u_{-}$since $F^{\prime}\left(u_{-}\right)=-\vec{n} \circ\left(f_{1}^{\prime}\left(u_{-}\right), f_{2}^{\prime}\left(u_{-}\right)\right)<$ $-\vec{n} \circ\left(f_{1}^{\prime}\left(u_{+}\right), f_{2}^{\prime}\left(u_{+}\right)\right)=F^{\prime}(\gamma u) \leq 0$ (see also Figure 5(b)) For $\forall k \in\left[u_{-}, \gamma u\right]$, according to the convex property of $F(u)$ and Lagrange mean value theorem, there exists $\xi \in[k, \gamma u]$, satisfying

$$
\frac{F(\gamma u)-F(k)}{\gamma u-k}=F^{\prime}(\xi) \leq F^{\prime}(\gamma u) \leq 0,
$$

and so the boundary entropy condition (4.10) is verified. 
In summary, we have the following theorem.

Theorem 4.1. Suppose that (\&) holds. Given $u_{-}, u_{+} \in(a, b)$, then

(i) if $u_{-}>u_{+}$and $\vec{n} \circ\left(\left[f_{1}\right] /[u],\left[f_{2}\right] /[u]\right) \geq 0$, the solution of (1.1) is constant state and has form as (4.2),

(ii) if $u_{-}>u_{+}$and $\vec{n} \circ\left(\left[f_{1}\right] /[u],\left[f_{2}\right] /[u]\right)<0$, the solution of $(1.1)$ is shock wave $\mathcal{S}$, and has form as (4.3),

(iii) if $u_{-}<u_{+}$and $\vec{n} \circ\left(f_{1}^{\prime}\left(u_{+}\right), f_{2}^{\prime}\left(u_{+}\right)\right)<\vec{n} \circ\left(f_{1}^{\prime}\left(u_{-}\right), f_{2}^{\prime}\left(u_{-}\right)\right) \leq 0$, the solution of (1.1) is rarefaction wave $R$ and has a form as (4.7),

(iv) if $u_{-}<u_{+}$and $\vec{n} \circ\left(f_{1}^{\prime}\left(u_{+}\right), f_{2}^{\prime}\left(u_{+}\right)\right)<0<\vec{n} \circ\left(f_{1}^{\prime}\left(u_{-}\right), f_{2}^{\prime}\left(u_{-}\right)\right)$, the solution of (1.1) is rarefaction wave $R$ and has a form as (4.8);

(v) if $u_{-}<u_{+}$and $0 \leq \vec{n} \circ\left(f_{1}^{\prime}\left(u_{+}\right), f_{2}^{\prime}\left(u_{+}\right)\right)<\vec{n} \circ\left(f_{1}^{\prime}\left(u_{-}\right), f_{2}^{\prime}\left(u_{-}\right)\right)$, the solution of (1.1) is constant state and has a form as (4.9).

In addition the solutions formed as (4.2), (4.3), (4.7), (4.8), and (4.9) uniquely exist.

Corollary 4.2. Suppose that $M_{x_{1}} f_{1}^{\prime \prime}(u)+M_{x_{2}} f_{2}^{\prime \prime}(u)<0$ for $u \in(a, b) . a, b$ can be finite or $\infty$, and when $u_{-}, u_{+} \in(a, b)$,

(i) if $u_{-}>u_{+}$and $\vec{n} \circ\left(f_{1}^{\prime}\left(u_{+}\right), f_{2}^{\prime}\left(u_{+}\right)\right)<\vec{n} \circ\left(f_{1}^{\prime}\left(u_{-}\right), f_{2}^{\prime}\left(u_{-}\right)\right) \leq 0$, the solution of (1.1) is rarefaction wave $R$ and has a form as (4.7),

(ii) if $u_{-}>u_{+}$and $\vec{n} \circ\left(f_{1}^{\prime}\left(u_{+}\right), f_{2}^{\prime}\left(u_{+}\right)\right)<0<\vec{n} \circ\left(f_{1}^{\prime}\left(u_{-}\right), f_{2}^{\prime}\left(u_{-}\right)\right)$, the solution of (1.1) is rarefaction wave $R$ and has a form as (4.8),

(iii) if $u_{-}>u_{+}$and $0 \leq \vec{n} \circ\left(f_{1}^{\prime}\left(u_{+}\right), f_{2}^{\prime}\left(u_{+}\right)\right)<\vec{n} \circ\left(f_{1}^{\prime}\left(u_{-}\right), f_{2}^{\prime}\left(u_{-}\right)\right)$, the solution of (1.1) is constant state and has a form as (4.9),

(iv) if $u_{-}<u_{+}$and $\vec{n} \circ\left(\left[f_{1}\right] /[u],\left[f_{2}\right] /[u]\right) \geq 0$, the solution of $(1.1)$ is constant state and has a form as (4.2),

(v) if $u_{-}<u_{+}$and $\vec{n} \circ\left(\left[f_{1}\right] /[u],\left[f_{2}\right] /[u]\right)<0$, the solution of $(1.1)$ is shock wave $\mathcal{S}$ and has a form as (4.3).

Corollary 4.3. The approach here for two-dimensional Riemann initial-boundary problem can be expanded to the case of general $n$-dimension.

\section{An Example}

Solve the following Riemann initial-boundary problem:

$$
\begin{gathered}
u_{t}+\left(\frac{u^{2}}{2}\right)_{x_{1}}+\left(\frac{u^{2}}{2}\right)_{x_{2}}=0, \quad\left(x_{1}, x_{2}\right) \in \Omega, t>0, \\
\left.u\right|_{t=0}=u_{+}, \quad\left(x_{1}, x_{2}\right) \in \Omega, \\
\left.u\right|_{\Gamma}=u_{-}, \quad t>0,
\end{gathered}
$$


where $M\left(x_{1}, x_{2}\right)=x_{1}^{3}+x_{2}, \Omega=\left\{\left(x_{1}, x_{2}\right) \mid M\left(x_{1}, x_{2}\right)>0\right\}, \Gamma=\left\{\left(t, x_{1}, x_{2}\right) \mid M\left(x_{1}, x_{2}\right)=\right.$ $0, t>0\}$, and it denotes $\left.u\right|_{\Gamma}=\gamma u$. Since $f_{1}(u)=f_{2}(u)=(1 / 2) u^{2}$, we easily get $M_{x_{1}} f_{1}^{\prime \prime}(u)+$ $M_{x_{2}} f_{2}^{\prime \prime}(u)=3 x_{1}^{2}+1>0$, and condition $\mathscr{H}$ holds.

According to the different data of $u_{+}$and $u_{-}$, the behavior of the solution to Riemann initial-boundary problem (5.1) has a total of five situations; they can be described by the following five cases: (i) $u_{-}=1, u_{+}=-2$; (ii) $u_{-}=2, u_{+}=-1$; (iii) $u_{-}=1, u_{+}=2$; (iv) $u_{-}=-1$, $u_{+}=1 ;(\mathrm{v}) u_{-}=-2, u_{+}=-1$.

For case (i), $u_{-}>u_{+}$and

$$
\begin{aligned}
\vec{n} \circ\left(\frac{\left[f_{1}\right]}{[u]}, \frac{\left[f_{2}\right]}{[u]}\right) & =-M_{x_{1}} \frac{\left[f_{1}\right]}{[u]}-M_{x_{2}} \frac{\left[f_{2}\right]}{[u]} \\
& =-3 x_{1}^{2} \frac{f_{1}\left(u_{-}\right)-f_{1}\left(u_{+}\right)}{u_{-}-u_{+}}-\frac{f_{2}\left(u_{-}\right)-f_{2}\left(u_{+}\right)}{u_{-}-u_{+}} \\
& =-\frac{1}{2}\left(3 x_{1}^{2}+1\right)\left(u_{-}+u_{+}\right) \\
& =\frac{1}{2}\left(3 x_{1}^{2}+1\right)>0,
\end{aligned}
$$

and thus the solution is constant state formed as

$$
u\left(t, x_{1}, x_{2}\right)=-2, \quad x_{1}^{3}+x_{2}>0, t>0 .
$$

For case (ii), $u_{-}>u_{+}$and

$$
\begin{aligned}
\vec{n} \circ\left(\frac{\left[f_{1}\right]}{[u]}, \frac{\left[f_{2}\right]}{[u]}\right) & =-M_{x_{1}} \frac{\left[f_{1}\right]}{[u]}-M_{x_{2}} \frac{\left[f_{2}\right]}{[u]} \\
& =-3 x_{1}^{2} \frac{f_{1}\left(u_{-}\right)-f_{1}\left(u_{+}\right)}{u_{-}-u_{+}}-\frac{f_{2}\left(u_{-}\right)-f_{2}\left(u_{+}\right)}{u_{-}-u_{+}} \\
& =-\frac{1}{2}\left(3 x_{1}^{2}+1\right)\left(u_{-}+u_{+}\right) \\
& =-\frac{1}{2}\left(3 x_{1}^{2}+1\right)<0,
\end{aligned}
$$

and thus the solution is shock wave solution formed as

$$
u\left(t, x_{1}, x_{2}\right)= \begin{cases}2, & x_{1}^{3}+x_{2}>0>\left(x_{1}-\frac{1}{2} t\right)^{3}+\left(x_{2}-\frac{1}{2} t\right), \quad t>0, \\ -1, & \left(x_{1}-\frac{1}{2} t\right)^{3}+\left(x_{2}-\frac{1}{2} t\right)>0, \quad t>0 .\end{cases}
$$


For case (iii), $u_{-}<u_{+}$and

$$
\begin{aligned}
& \vec{n} \circ\left(f_{1}^{\prime}\left(u_{-}\right), f_{2}^{\prime}\left(u_{-}\right)\right)=-M_{x_{1}} f_{1}^{\prime}\left(u_{-}\right)-M_{x_{2}} f_{2}^{\prime}\left(u_{-}\right)=-\left(3 x_{1}^{2}+1\right), \\
& \vec{n} \circ\left(f_{1}^{\prime}\left(u_{+}\right), f_{2}^{\prime}\left(u_{+}\right)\right)=-M_{x_{1}} f_{1}^{\prime}\left(u_{+}\right)-M_{x_{2}} f_{2}^{\prime}\left(u_{+}\right)=-2\left(3 x_{1}^{2}+1\right),
\end{aligned}
$$

namely, $\vec{n} \circ\left(f_{1}^{\prime}\left(u_{+}\right), f_{2}^{\prime}\left(u_{+}\right)\right)<\vec{n} \circ\left(f_{1}^{\prime}\left(u_{-}\right), f_{2}^{\prime}\left(u_{-}\right)\right) \leq 0$, thus the solution is rarefaction wave formed as

$$
u\left(t, x_{1}, x_{2}\right)= \begin{cases}1, & x_{1}^{3}+x_{2}>0>\left(x_{1}-t\right)^{3}+\left(x_{2}-t\right), \quad t>0 \\ C\left(t, x_{1}, x_{2}\right), & \left(x_{1}-t\right)^{3}+\left(x_{2}-t\right) \geq 0 \geq\left(x_{1}-2 t\right)^{3}+\left(x_{2}-2 t\right), \quad t>0 \\ 2, & \left(x_{1}-2 t\right)^{3}+\left(x_{2}-2 t\right)>0, \quad t>0 .\end{cases}
$$

Here, we only need to solve $C\left(t, x_{1}, x_{2}\right) \in \Omega_{C}$, where

$$
\Omega_{C}=\left\{\left(t, x_{1}, x_{2}\right) \mid\left(x_{1}-t\right)^{3}+\left(x_{2}-t\right) \geq 0 \geq\left(x_{1}-2 t\right)^{3}+\left(x_{2}-2 t\right), t>0\right\} .
$$

To solve the following equation of $C$ :

$$
M\left(x_{1}-f_{1}^{\prime}(C) t, x_{2}-f_{2}^{\prime}(C) t\right)=\left(x_{1}-C t\right)^{3}+\left(x_{2}-C t\right)=0,
$$

using Cardano formula, we can get the unique solution as

$$
\begin{aligned}
C\left(t, x_{1}, x_{2}\right)= & \frac{1}{\sqrt[3]{2} t}\left[\sqrt[3]{-\left(x_{1}-x_{2}\right)+\sqrt{\frac{4}{27}+\left(x_{1}-x_{2}\right)^{2}}}\right. \\
& \left.+\sqrt[3]{-\left(x_{1}-x_{2}\right)-\sqrt{\frac{4}{27}+\left(x_{1}-x_{2}\right)^{2}}}\right]+\frac{x_{1}}{t} .
\end{aligned}
$$

Since $C\left(t, x_{1}, x_{2}\right)$ is the solution of implicit function, we still need to verify $C\left(t, x_{1}, x_{2}\right)$ satisfying the following three conditions: (a) $C_{t}+C C_{x_{1}}+C C_{x_{2}}=0,\left(t, x_{1}, x_{2}\right) \in \Omega_{C}$; (b) $C=$ $1=u_{-},\left(x_{1}-t\right)^{3}+\left(x_{2}-t\right)=0, t>0$; (c) $C=2=u_{+},\left(x_{1}-2 t\right)^{3}+\left(x_{2}-2 t\right)=0, t>0$. In fact, according to the next proposition, the above three conditions can be easily verified, and the detail the omitted here.

Proposition 5.1. For any real number $x$, the following formula holds:

$$
\sqrt[3]{-\left(x+x^{3}\right)+\sqrt{\frac{4}{27}+\left(x+x^{3}\right)^{2}}}+\sqrt[3]{-\left(x+x^{3}\right)-\sqrt{\frac{4}{27}+\left(x+x^{3}\right)^{2}}}+\sqrt[3]{2} x=0 .
$$


Proof. Let

$$
p=\sqrt[3]{-\left(x+x^{3}\right)+\sqrt{\frac{4}{27}+\left(x+x^{3}\right)^{2}}}+\sqrt[3]{-\left(x+x^{3}\right)-\sqrt{\frac{4}{27}+\left(x+x^{3}\right)^{2}}},
$$

then $p$ satisfies

$$
E(p) \triangleq p^{3}+\sqrt[3]{4} p+2\left(x+x^{3}\right)=0
$$

where $p$ must be one root of (5.13). In fact, $E^{\prime}(p)=3 p^{2}+\sqrt[3]{4}>0$. Equation (5.13) at most has one real root; but $-\sqrt[3]{2} x$ is its real root, thus $p=-\sqrt[3]{2} x$, and the proposition holds.

For case (iv), $u_{-}<u_{+}$and

$$
\begin{gathered}
\vec{n} \circ\left(f_{1}^{\prime}\left(u_{-}\right), f_{2}^{\prime}\left(u_{-}\right)\right)=-M_{x_{1}} f_{1}^{\prime}\left(u_{-}\right)-M_{x_{2}} f_{2}^{\prime}\left(u_{-}\right)=3 x_{1}^{2}+1, \\
\vec{n} \circ\left(f_{1}^{\prime}\left(u_{+}\right), f_{2}^{\prime}\left(u_{+}\right)\right)=-M_{x_{1}} f_{1}^{\prime}\left(u_{+}\right)-M_{x_{2}} f_{2}^{\prime}\left(u_{+}\right)=-\left(3 x_{1}^{2}+1\right),
\end{gathered}
$$

namely, $\vec{n} \circ\left(f_{1}^{\prime}\left(u_{+}\right), f_{2}^{\prime}\left(u_{+}\right)\right)<0<\vec{n} \circ\left(f_{1}^{\prime}\left(u_{-}\right), f_{2}^{\prime}\left(u_{-}\right)\right)$, and thus the solution is rarefaction wave formed as

$$
u\left(t, x_{1}, x_{2}\right)= \begin{cases}C\left(t, x_{1}, x_{2}\right), & x_{1}^{3}+x_{2}>0 \geq\left(x_{1}-t\right)^{3}+\left(x_{2}-t\right), \quad t>0 \\ 1, & \left(x_{1}-t\right)^{3}+\left(x_{2}-t\right)>0, \quad t>0,\end{cases}
$$

where $C\left(t, x_{1}, x_{2}\right)$ has the same form as (5.10).

For case $(\mathrm{v}), u_{-}<u_{+}$and

$$
\begin{gathered}
\vec{n} \circ\left(f_{1}^{\prime}\left(u_{-}\right), f_{2}^{\prime}\left(u_{-}\right)\right)=-M_{x_{1}} f_{1}^{\prime}\left(u_{-}\right)-M_{x_{2}} f_{2}^{\prime}\left(u_{-}\right)=2\left(3 x_{1}^{2}+1\right), \\
\vec{n} \circ\left(f_{1}^{\prime}\left(u_{+}\right), f_{2}^{\prime}\left(u_{+}\right)\right)=-M_{x_{1}} f_{1}^{\prime}\left(u_{+}\right)-M_{x_{2}} f_{2}^{\prime}\left(u_{+}\right)=3 x_{1}^{2}+1,
\end{gathered}
$$

namely, $0 \leq \vec{n} \circ\left(f_{1}^{\prime}\left(u_{+}\right), f_{2}^{\prime}\left(u_{+}\right)\right)<\vec{n} \circ\left(f_{1}^{\prime}\left(u_{-}\right), f_{2}^{\prime}\left(u_{-}\right)\right)$, and thus the solution is constant state formed as

$$
u\left(t, x_{1}, x_{2}\right)=-1, \quad x_{1}^{3}+x_{2}>0, t>0
$$

\section{Acknowledgment}

This work is supported by the National Natural Science Foundation of China (10771087, 61078040), the Natural Science Foundation of Guangdong Province (7005948). 


\section{References}

[1] S. N. Kruzkov, "Generalized solutions of the Cauchy problem in the large for nonlinear equations of first order," Soviet Mathematics Doklady, vol. 10, pp. 785-788, 1969.

[2] C. Bardos, A. Y. le Roux, and J.-C. Nédélec, "First order quasilinear equations with boundary conditions," Communications in Partial Differential Equations, vol. 4, no. 9, pp. 1017-1034, 1979.

[3] P. A. Razafimandimby and M. Sango, "Weak solutions of a stochastic model for two-dimensional second grade fluids," Boundary Value Problems, vol. 2010, Article ID 636140, 47 pages, 2010.

[4] E. Conway and J. Smoller, "Clobal solutions of the Cauchy problem for quasi-linear first-order equations in several space variables," Communications on Pure and Applied Mathematics, vol. 19, pp. 95-105, 1966.

[5] W. B. Lindquist, "The scalar Riemann problem in two spatial dimensions: piecewise smoothness of solutions and its breakdown," SIAM Journal on Mathematical Analysis, vol. 17, no. 5, pp. 1178-1197, 1986.

[6] D. H. Wagner, "The Riemann problem in two space dimensions for a single conservation law," SIAM Journal on Mathematical Analysis, vol. 14, no. 3, pp. 534-559, 1983.

[7] T. Zhang and Y. X. Zheng, "Two-dimensional Riemann problem for a single conservation law," Transactions of the American Mathematical Society, vol. 312, no. 2, pp. 589-619, 1989.

[8] J. Guckenheimer, "Shocks and rarefactions in two space dimensions," Archive for Rational Mechanics and Analysis, vol. 59, no. 3, pp. 281-291, 1975.

[9] Y. Zheng, Systems of Conservation Laws: Two-Dimensional Riemann Problems, vol. 38 of Progress in Nonlinear Differential Equations and Their Applications, Birkhäuser, Boston, Mass, USA, 2001.

[10] X. Yang, "Multi-dimensional Riemann problem of scalar conservation law," Acta Mathematica Scientia, vol. 19, no. 2, pp. 190-200, 1999.

[11] F. Dubois and P. LeFloch, "Boundary conditions for nonlinear hyperbolic systems of conservation laws," Journal of Differential Equations, vol. 71, no. 1, pp. 93-122, 1988.

[12] P. LeFloch and J.-C. Nédélec, "Explicit formula for weighted scalar nonlinear hyperbolic conservation laws," Transactions of the American Mathematical Society, vol. 308, no. 2, pp. 667-683, 1988.

[13] T. Pan and L. Lin, "The global solution of the scalar nonconvex conservation law with boundary condition," Journal of Partial Differential Equations, vol. 8, no. 4, pp. 371-383, 1995.

[14] T. Pan and L. Lin, "The global solution of the scalar nonconvex conservation law with boundary condition. (Continuation)," Journal of Partial Differential Equations, vol. 11, no. 1, pp. 1-8, 1998.

[15] T. Pan, H. Liu, and K. Nishihara, "Asymptotic stability of the rarefaction wave of a one-dimensional model system for compressible viscous gas with boundary," Japan Journal of Industrial and Applied Mathematics, vol. 16, no. 3, pp. 431-441, 1999.

[16] T. Pan and Q. Jiu, "Asymptotic behaviors of the solutions to scalar viscous conservation laws on bounded interval corresponding to rarefaction waves," Progress in Natural Science, vol. 9, no. 12, pp. 948-952, 1999.

[17] T. Pan and H. Liu, "Asymptotic behaviors of the solution to an initial-boundary value problem for scalar viscous conservation laws," Applied Mathematics Letters, vol. 15, no. 6, pp. 727-734, 2002.

[18] T. Pan, H. Liu, and K. Nishihara, "Asymptotic behavior of a one-dimensional compressible viscous gas with free boundary," SIAM Journal on Mathematical Analysis, vol. 34, no. 2, pp. 273-291, 2002. 